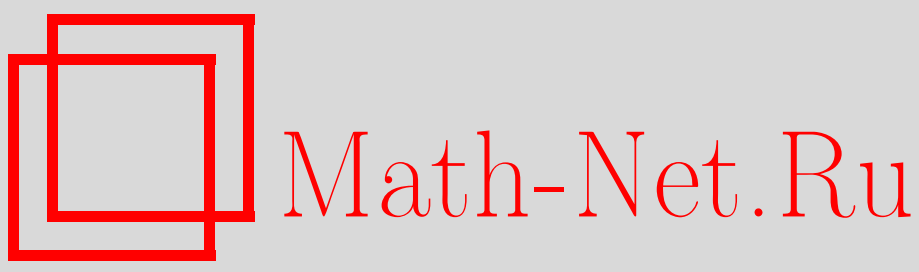

В. П. Чистяков, Об асимптотической нормальности числа пустых ячеек в одной схеме размещения частиц комплектами, Матем. вопр. криптогр., 2014, том 5, выпуск 4, 129-138

DOI: https://doi.org/10.4213/mvk138

Использование Общероссийского математического портала Math-Net.Ru подразумевает, что вы прочитали и согласны с пользовательским соглашением

http://www.mathnet.ru/rus/agreement

Параметры загрузки:

IP: 34.239 .49 .27

26 апреля 2023 г., 10:32:55

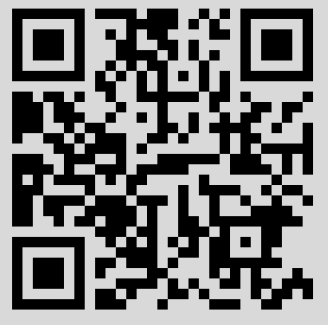


МАТЕМАТИЧЕСКИЕ ВОПРОСЫ КРИПТОГРАФИИ

2014 T. 5 № 4 C. $129-138$

УДК 519.212.2+519.214.5

\title{
Об асимптотической нормальности числа пустых ячеек в одной схеме размещения частиц комплектами
}

\author{
В. П. Чистяков \\ Математический институт им.В.А.Стеклова РАН, Москва
}

Получено 18.III.2013

Рассматривается схема $m$-зависимого размещения по ячейкам неравновероятных комплектов частиц. Предполагается, что число комплектов и число ячеек стремятся к бесконечности и имеют одинаковый порядок. Получены асимптотические формулы для математического ожидания и дисперсии, а также достаточные условия асимптотической нормальности числа пустых ячеек в этой схеме.

Ключевые слова: $m$-зависимые последовательности, размещения комплектами, асимптотическая нормальность, число пустых ячеек.

On the asymptotic normality in a scheme of allocation of nonequiprobable particle sets V.P. Chistyakov

Steklov Mathematical Institute of RAS, Moscow

Abstract. A scheme of allocation of non-equiprobable $m$-dependent particle sets into cells is considered. The number of cells and the number of sets of particles are supposed to tend to $\infty$ and to have the same order of growth. Asymptotic formulas for mathematical expectation and variance of the number of empty cells are obtained along with the sufficient conditions for their asymptotic normality.

Keywords: $m$-dependent sequences, allocations of sets, asymptotic normality, a number of empty cells

Citation: Mathematical Aspects of Cryptography, 2014, vol. 5, no. 4, pp. 129-138 (Russian) (C) 2014 В. П. Чистяков 


\section{1. Постановка задачи}

В классической постановке задачи о размещении частиц комплектами считается, что имеется $N$ ячеек, в которые бросают независимо $n$ комплектов по $v$ частиц в каждом. При этом предполагается, что частицы одного комплекта попадают в разные ячейки и все $C_{N}^{v}$ размещений частиц каждого комплекта равновероятны. Эта схема рассматривалась многими авторами (см. [1], гл. VII; [2]). Рассматривались также различные обобщения равновероятной схемы: равновероятная схема с комплектами неодинаковых размеров ([11]), схемы независимого размещения неравновероятных комплектов (см., например, [3]) и т. д. Схемы независимого размещения комплектов случайного объема использовались при исследовании числа решений некоторых систем случайных уравнений $([9,10])$.

Рассмотрим следующую схему $m$-зависимого размещения частиц комплектами. Обозначим через $\mathcal{M}$ множество всех $v$-элементных подмножеств множества $\mathcal{N}=\{1,2, \ldots, N\}$ :

$$
\mathcal{M}=\left\{I=\left(i_{1}, \ldots, i_{v}\right): 1 \leqslant i_{1}<i_{2}<\cdots<i_{v} \leqslant N\right\} .
$$

Пусть

$$
Z_{1}, Z_{2}, \ldots, Z_{t}, \ldots \quad, \quad Z_{t} \in \mathcal{M}
$$

- такая стационарная последовательность $m$-зависимых случайных подмножеств $I \in \mathcal{M}$, что наборы $\left(Z_{s-u_{1}}, \ldots, Z_{s}\right)$ и $\left(Z_{t}, \ldots, Z_{t+u_{2}}\right)$ независимы при любых $u_{1}, u_{2}, s, t$, для которых $t-s>m$.

Случайная величина $\mu_{0}=\mu_{0}(n, N)$, равная числу ячеек, оставшихся пустыми после размещения по $N$ ячейкам частиц всех $n$ комплектов, выражается равенством

$$
\mu_{0}=\sum_{i=1}^{N} \chi\left(Z_{1} \notin K_{i}, Z_{2} \notin K_{i}, \ldots, Z_{n} \notin K_{i}\right),
$$

где $\chi(C)$ - индикатор события $C, K_{i}=\{I: I \in \mathcal{M}, i \in I\}$. В данной работе получены достаточные условия асимптотической нормальности $\mu_{0}(n, N)$ и асимптотические формулы для $\mathbf{E} \mu_{0}, \mathbf{E} \mu_{0}^{[2]}\left(\mu_{0}^{[2]}=\mu_{0}\left(\mu_{0}-1\right)\right)$, $\mathbf{D} \mu_{0}$ в предположении, что $n, N \rightarrow \infty$,

$$
\frac{n}{N}=\alpha_{N}, \quad 0<\underline{\alpha} \leqslant \alpha_{N} \leqslant \bar{\alpha}<\infty
$$

и совместное распределение случайных множеств $Z_{1}, \ldots, Z_{n}$ меняется так, что при любых $t_{1}<\cdots<t_{l}$ и любых $I_{1}, \ldots, I_{l} \in \mathcal{M}$

$$
0<\underline{c}^{l} \leqslant M^{l} \mathbf{P}\left(Z_{t_{1}}=I_{1}, \ldots, Z_{t_{l}}=I_{l}\right) \leqslant \bar{c}^{l}, \quad l=1,2, \ldots,
$$


где $\underline{\alpha}, \bar{\alpha}, \underline{c}, \bar{c}$ - некоторые постоянные, $M=|\mathcal{M}|=C_{N}^{v}$.

Приведем пример $m$-зависимых величин $(1)$, для которых выполняется условие (3). Пусть

$$
\zeta_{1}, \zeta_{2}, \ldots, \zeta_{t}, \ldots
$$

- стационарная последовательность независимых случайных элементов множества $\mathcal{M}$. Перенумеруем каким-либо образом элементы множества $\mathcal{M}:$

$$
\mathcal{M}=\left\{I=\left(i_{1}, \ldots, i_{v}\right): 1 \leqslant i_{1}<\cdots<i_{v} \leqslant N\right\}=\left\{I_{0}, I_{1}, \ldots, I_{M-1}\right\} .
$$

Положим

$$
\mathbf{P}\left\{\zeta_{t}=I_{u}\right\}=p_{u}, \quad u=0,1, \ldots, M-1 .
$$

Суммой $I_{k_{1}} \oplus I_{k_{2}}$ двух элементов $I_{k_{1}}$ и $I_{k_{2}}$ назовем элемент $I_{k}$, где $k=$ $k_{1}+k_{2} \bmod M$. Тогда последовательность

$$
Z_{t}=\zeta_{t} \oplus \zeta_{t+1} \oplus \cdots \oplus \zeta_{t+m}, \quad t=1,2, \ldots
$$

является последовательностью $m$-зависимых множеств. Если

$$
0<c_{0} \leqslant M p_{u} \leqslant c_{1}<\infty, \quad u=0,1, \ldots, M-1,
$$

где $c_{0}, c_{1}$ - некоторые постоянные, то для последовательности (5) выполняется условие (3).

\section{2. Асимптотические формулы для моментов}

Покажем, что неравенства вида (3) выполняются для последовательностей

$$
\chi_{i}(1), \ldots, \chi_{i}(n) ; \quad \chi_{i j}(1), \ldots, \chi_{i j}(n), \quad i, j \in \mathcal{N},
$$

где

$$
\chi_{i j}(t)=\chi\left(Z_{t} \in K_{i} \cup K_{j}\right) .
$$

Лемма 1. Если выполняется условие (3), то при любых $t_{1}, \ldots, t_{l}$ и любыx $i, j \in \mathcal{N}$

$$
0<\underline{c}_{1}^{l} \leqslant N^{l} \mathbf{E} \chi_{i}\left(t_{1}\right) \ldots \chi_{i}\left(t_{l}\right) \leqslant \bar{c}_{1}^{l}, \quad 0<\underline{c}_{2}^{l} \leqslant N^{l} \mathbf{E} \chi_{i j}\left(t_{1}\right) \ldots \chi_{i j}\left(t_{l}\right) \leqslant \bar{c}_{2}^{l},
$$

где $l=1,2, \ldots, a \underline{c}_{1}, \bar{c}_{1}, \underline{c}_{2}, \bar{c}_{2}$ - некоторые постоянные.

Доказательство. Для элементов множеств $K_{i}$ и $K_{i} \cup K_{j}$ введем обозначения

$$
K_{i}=\left\{I_{1}^{(i)}, \ldots, I_{\left|K_{i}\right|}^{(i)}\right\}, \quad K_{i} \cup K_{j}=\left\{I_{1}^{(i, j)}, \ldots, I_{\left|K_{i} \cup K_{j}\right|}^{(i, j)}\right\}
$$


Нетрудно проверить, что

$$
\left|K_{i}\right|=C_{N-1}^{v-1},\left|K_{i} \cap K_{j}\right|=C_{N-2}^{v-2},\left|K_{i} \cup K_{j}\right|=2 C_{N-1}^{v-1}-C_{N-2}^{v-2}
$$

и

$$
\begin{gathered}
\mathbf{E} \chi_{i}\left(t_{1}\right) \ldots \chi_{i}\left(t_{l}\right)= \\
=\sum_{u_{1}, \ldots, u_{l}=1}^{\left|K_{i}\right|} \mathbf{P}\left(Z_{t_{1}}=I_{u_{1}}^{(i)}, \ldots, Z_{t_{l}}=I_{u_{l}}^{(i)}\right) \leqslant\left|K_{i}\right|^{l}\left(\frac{\bar{c}}{M}\right)^{l}=\frac{(v \bar{c})^{l}}{N^{l}}, \\
\mathbf{E} \chi_{i j}\left(t_{1}\right) \ldots \chi_{i j}\left(t_{l}\right)=\sum_{u_{1}, \ldots, u_{l}=1}^{\left|K_{i} \cup K_{j}\right|} \mathbf{P}\left(Z_{t_{1}}=I_{u_{1}}^{(i, j)}, \ldots, Z_{t_{l}}=I_{u_{l}}^{(i, j)}\right) \leq \\
\leqslant\left|K_{i} \cup K_{j}\right|^{l}\left(\frac{\bar{c}}{M}\right)^{l} \leqslant \frac{(2 v \bar{c})^{l}}{N^{l}} .
\end{gathered}
$$

Аналогично получаются нижние оценки.

Введем обозначения

$$
\begin{aligned}
& a_{i}=N \mathbf{E} \chi_{i}(t), \quad a_{i j}=N \mathbf{E} \chi_{i j}(t), \quad a_{i j}^{*}=N^{2} \mathbf{P}\left(Z_{t} \in K_{i} \cap K_{j}\right), \\
& b_{i j}(u)=N^{2} \mathbf{E} \chi_{i}(t) \chi_{j}(t+u), \quad b_{i j}=\sum_{u=1}^{m} b_{i j}(u), \\
& B_{i j}(u)=N^{2} \mathbf{E} \chi_{i j}(t) \chi_{i j}(t+u), B_{i j}=\sum_{u=1}^{m} B_{i j}(u) .
\end{aligned}
$$

Лемма 2. В схеме серий (2) - (3) при любых $i, j \in \mathcal{N}, \quad u=1, \ldots, m$,

$$
\underline{a} \leqslant a_{i} \leqslant \bar{a}, \quad \underline{a} \leqslant a_{i j} \leqslant \bar{a}, \quad \underline{a} \leqslant b_{i j} \leqslant \bar{a},
$$

$a_{i j}=a_{i}+a_{j}-\frac{1}{N} a_{i j}^{*}, \quad B_{i j}(u)=b_{i i}(u)+b_{i j}(u)+b_{j i}(u)+b_{j j}(u)+O\left(\frac{1}{N}\right)$,

$$
B_{i j}=b_{i i}+b_{i j}+b_{j i}+b_{j j}+O\left(\frac{1}{N}\right)
$$

где $\bar{a}, \underline{a}, 0<\underline{a}<\bar{a},-$ некоторые постоянные.

Доказательство. Неравенства (8) следуют из леммы 1. Из равенства

$$
\mathbf{E} \chi_{i j}(t)=\mathbf{E} \chi_{i}(t)+E \chi_{j}(t)-\mathbf{P}\left(Z_{t} \in K_{i} \cap K_{j}\right)
$$


следует равенство $(9)$ для $a_{i j}$.

Представим $\chi_{i j}(t) \chi_{i j}(t+u)$ в виде

$$
\mathbf{E} \chi_{i j}(t) \chi_{i j}(t+u)=\mathbf{P}\left(D_{1}\right)+\mathbf{P}\left(D_{2}\right)-\mathbf{P}\left(D_{1} \cap D_{2} \cap D\right),
$$

где $D=\left(Z_{t+u} \in K_{i}\right) \cup\left(Z_{t+u} \in K_{j}\right)$,

$$
D_{1}=\left(Z_{t} \in K_{i}\right) \cap D, \quad D_{2}=\left(Z_{t} \in K_{j}\right) \cap D .
$$

Аналогично доказательству леммы 1 получим

$$
\mathbf{P}\left(Z_{t} \in K_{i} \cap K_{j} \cap D\right) \leqslant \frac{C}{N^{3}},
$$

где $C>0-$ некоторая постоянная. Вероятность $\mathbf{P}\left(D_{1}\right)$ представим в виде

$$
\begin{gathered}
\mathbf{P}\left(D_{1}\right)=\mathbf{P}\left(Z_{t} \in K_{i}, Z_{t+u} \in K_{i}\right)+\mathbf{P}\left(Z_{t} \in K_{i}, Z_{t+u} \in K_{j}\right)- \\
-\mathbf{P}\left(\left(Z_{t} \in K_{i}\right) \cap\left(Z_{t+u} \in K_{i} \cap K_{j}\right)\right) .
\end{gathered}
$$

Отсюда

$$
\mathbf{P}\left(D_{1}\right)=\frac{b_{i i}(u)}{N^{2}}+\frac{b_{i j}(u)}{N^{2}}+O\left(\frac{1}{N^{3}}\right) .
$$

Точно так же оценивается вероятность $\mathbf{P}\left(D_{2}\right)$.

Из полученных равенств следует утверждение для $B_{i j}(u)$ и $B_{i j}$. Лемма доказана.

Теорема 1. При $n, N \rightarrow \infty$ и некотором $\delta, 0<\delta<1$, в схеме серий $(2)-(3)$

$$
\begin{gathered}
\mathbf{E} \mu_{0}(n, N)=N M_{0}-\frac{1}{2} \alpha_{N}(2 m+1) M_{2}+\alpha_{N} M^{*}+O\left(\frac{1}{N^{\delta}}\right) \\
\mathbf{E} \mu_{0}^{[2]}(n, N)=N^{2} M_{0}-N\left(\frac{1}{N} \sum_{i=1}^{N} e^{-2 \alpha_{N} a_{i}}\right)- \\
-N \alpha_{N}(2 m+1)\left(M_{0} M_{2}+M_{1}^{2}\right)+N \alpha_{N}\left(\frac{1}{N^{2}} \sum_{i, j=1}^{N} a_{i j}^{*} e^{-\alpha_{N}\left(a_{i}+a_{j}\right)}\right)+ \\
+2 \alpha_{N} N\left(M_{0} M^{*}+\frac{1}{N^{2}} \sum_{i, j=1}^{N} b_{i j} e^{-\alpha_{N}\left(a_{i}+a_{j}\right)}\right)+O\left(N^{\delta}\right),
\end{gathered}
$$

где $a_{i}, b_{i j}, a_{i j}^{*}$ определены равенствами (7),

$$
M_{k}=\frac{1}{N} \sum_{i=1}^{N} a_{i}^{k} e^{-\alpha_{N} a_{i}}, \quad k=0,1,2, \quad M^{*}=\frac{1}{N} \sum_{i=1}^{N} b_{i i} e^{-\alpha_{N} a_{i}} .
$$


Доказательство теоремы. Используя равенство

$$
\mu_{0}=\sum_{i=1}^{N}\left(1-\chi_{i}(1)\right)\left(1-\chi_{i}(2)\right) \ldots\left(1-\chi_{i}(n)\right),
$$

получаем

$$
\mu_{0}(n, N)=\sum_{l=0}^{n} \sum_{t(l) \in T(l)} \xi_{l}(t(l))
$$

где

$$
\begin{aligned}
& \chi_{i}(t)=\chi\left(Z_{t} \in A_{i}\right), \quad t(l)=\left(t_{1}, \ldots, t_{l}\right), \\
& T(l)=\left\{t(l): 1 \leqslant t_{1}<\cdots<t_{l} \leqslant n\right\}, \quad l \geqslant 1, \\
& \xi_{l}(t(l))=(-1)^{l} \sum_{i=1}^{N} \chi\left(Z_{t_{1}} \in A_{i}, \ldots, Z_{t_{l}} \in A_{i}\right) .
\end{aligned}
$$

Так же, как и в работе [4], используя формулы включенияисключения, представим $\mathbf{E} \mu_{0}, \mathbf{E} \mu_{0}^{[2]}$ в виде

$$
\mathbf{E} \mu_{0}=\sum_{i=1}^{N} P_{i}, \quad \mathbf{E} \mu_{0}^{[2]}=\sum_{i \neq j} P_{i j},
$$

где при любом $\varphi, 1 \leq \varphi<N$,

$$
\begin{aligned}
& P_{i}=P_{i}(\varphi)+Q_{i}(\varphi), P_{i j}=P_{i j}(\varphi)+Q_{i j}(\varphi), \\
& P_{i}(\varphi)=\sum_{l=0}^{\varphi-1}(-1)^{l} S_{l}(i), \quad P_{i j}(\varphi)=\sum_{l=0}^{\varphi-1}(-1)^{l} S_{l}(i, j), \\
& S_{l}(i)=\sum_{t(l) \in T(l)} \mathbf{E}\left(\chi_{i}\left(t_{1}\right) \ldots \chi_{i}\left(t_{l}\right)\right), \quad l \geqslant 1, \quad S_{0}(i)=1, \\
& S_{l}(i, j)=\sum_{t(l) \in T(l)} \mathbf{E}\left(\chi_{i j}\left(t_{1}\right) \ldots \chi_{i j}\left(t_{l}\right)\right), \quad S_{0}(i, j)=1, \quad i \neq j, \\
& \left|Q_{i}(\varphi)\right| \leqslant S_{\varphi}(i), \quad\left|Q_{i j}(\varphi)\right| \leqslant S_{\varphi}(i, j) .
\end{aligned}
$$

Из этих формул получим следующие представления для моментов:

$$
\mathbf{E} \mu_{0}=\sum_{l=0}^{\varphi-1}(-1)^{l} A_{l}+\tilde{A}_{n}, \quad \mathbf{E} \mu_{0}^{[2]}=\sum_{l=0}^{\varphi-1}(-1)^{l} B_{l}+\tilde{B}_{n},
$$


где

$$
A_{l}=\sum_{i=1}^{N} S_{l}(i), B_{l}=\sum_{i \neq j} S_{l}(i, j), \quad\left|\tilde{A}_{n}\right| \leqslant A_{\varphi}, \quad\left|\tilde{B}_{n}\right| \leqslant B_{\varphi}
$$

Положим

$$
\tau(t)=(t, t+1, \ldots, t+m) .
$$

Множество $T(l)$, определяемое равенством (2), представим в виде объединения трех не пересекающихся множеств:

$$
T(l)=T_{1}(l) \cup T_{2}(l) \cup T_{3}(l),
$$

где

$T_{1}(l)=\left\{t(l): \tau\left(t_{u_{1}}\right) \cap \tau\left(t_{u_{2}}\right)=\varnothing\right.$ при любых $\left.u_{1} \neq u_{2}, u_{1}, u_{2}=1, \ldots, l\right\}$, $T_{2}(l)=\left\{t(l)\right.$ : среди $\tau\left(t_{1}\right), \ldots, \tau\left(t_{l}\right)$ ровно два множества пересекаются $\}$, $T_{3}(l)=T(l) \backslash\left(T_{1}(l) \cup T_{2}(l)\right)$.

Так же, как в работах [4], [5] разобьем суммы $S_{l}(i), S_{l}(i, j)$ на три части, $S_{l}(i)=S_{l}^{(1)}(i)+S_{l}^{(2)}(i)+S_{l}^{(3)}(i), \quad S_{l}(i, j)=S_{l}^{(1)}(i, j)+S_{l}^{(2)}(i, j)+S_{l}^{(3)}(i, j)$, соответствующие разбиениям (19). Отметим, что

$$
S_{0}^{(1)}(i)=S_{0}^{(1)}(i, j)=1, \quad S_{0}^{(u)}(i)=S_{0}^{(u)}(i, j)=0, \quad u=2,3 .
$$

Подставив в (18) эти разбиения сумм, представим величины $A_{l}, B_{l}$ в виде сумм трех слагаемых каждую:

$$
A_{l}=A_{l}^{(1)}+A_{l}^{(2)}+A_{l}^{(3)}, \quad B_{l}=B_{l}^{(1)}+B_{l}^{(2)}+B_{l}^{(3)} .
$$

Дальнейшие рассуждения проводятся аналогично доказательствам из работ [4], [5]. Отметим изменения, которые нужно внести в доказательства асимптотических формул, приведенных в работе [5]. Необходимо оценить величины

$$
\begin{aligned}
& \mathbf{E} \chi_{i}\left(t_{1}\right) \ldots \chi_{i}\left(t_{l}\right)=\mathbf{P}\left(Z_{t_{1}} \in K_{i}, \ldots, Z_{t_{l}} \in K_{i}\right), \\
& \mathbf{E} \chi_{i j}\left(t_{1}\right) \ldots \chi_{i j}\left(t_{l}\right)=\mathbf{P}\left(Z_{t_{1}} \in K_{i} \cup K_{j}, \ldots, Z_{t_{l}} \in K_{i} \cup K_{j}\right),
\end{aligned}
$$

которые входят в суммы $(16)$. Если $t(l) \in T_{1}(l)$, то

$$
\begin{aligned}
& \mathbf{E} \chi_{i}\left(t_{1}\right) \ldots \chi_{i}\left(t_{l}\right)=\left(\frac{a_{i}}{N}\right)^{l}, \\
& \mathbf{E} \chi_{i j}\left(t_{1}\right) \ldots \chi_{i j}\left(t_{l}\right)=\left(\frac{a_{i}+a_{j}-\frac{1}{N} a_{i j}^{*}}{N}\right)^{l} .
\end{aligned}
$$


При

$$
\begin{gathered}
t(l) \in T_{2}^{(u)}(l, k, n)= \\
=\left\{t(l): t(l) \in T_{l}, \quad t_{k+1}-t_{k}=u, \quad t_{k_{1}+1}-t_{k_{1}} \geqslant m+1, \quad k_{1} \neq k\right\}
\end{gathered}
$$

нужно воспользоваться следующими из леммы 2 формулами

$$
\begin{aligned}
& \mathbf{E} \chi_{i}(t) \chi_{i}(t+u)=\frac{1}{N^{2}} b_{i}(u), \\
& \mathbf{E} \chi_{i j}(t) \chi_{i j}(t+u)=\frac{1}{N^{2}}\left(b_{i i}(u)+b_{i j}(u)+b_{j i}(u)+b_{j j}(u)+O(1 / N)\right),
\end{aligned}
$$

$u=1, \ldots, m, \quad i, j \in \mathcal{N}$. В случае $t(l) \in T_{3}(l)$ нужно воспользоваться леммой 1. Остальные выкладки мы опускаем. Теорема доказана.

Из теоремы 1 получаем следующее утверждение.

Следствие 1. При $n, N \rightarrow \infty$ в схеме серий (2) - (3)

$$
\mathbf{D} \mu_{0}(n, N)=N \sigma_{N}^{2}\left(\alpha_{N}\right)+O\left(N^{\delta}\right), \quad 0<\delta<1,
$$

әде

$$
\begin{array}{r}
\sigma_{N}^{2}\left(\alpha_{N}\right)=M_{0}-\frac{1}{N} \sum_{i=1}^{N} e^{-2 \alpha_{N} a_{i}}-\alpha_{N} M_{1}^{2}+\alpha_{N}\left(\frac{1}{N^{2}} \sum_{i, j=1}^{N} a_{i j}^{*} e^{-\alpha_{N}\left(a_{i}+a_{j}\right)}\right)+ \\
+2 \alpha_{N}\left(\frac{1}{N^{2}} \sum_{i, j=1}^{N} e^{-\alpha_{N}\left(a_{i}+a_{j}\right)}\left(b_{i j}-m a_{i} a_{j}\right)\right)
\end{array}
$$

а величины $a_{i}, a_{i j}^{*}, b_{i j}, M_{0}, M_{1}$ определены равенствами (7), (11).

Если объём комплекта $v=1$, то $a_{i j}^{*}=0$, и из этой формулы для $\sigma_{N}^{2}\left(\alpha_{N}\right)$ получается формула (30) работы [5].

Для схемы независимых и равновероятных комплектов из [1], используя равенства $b_{i j}=m a_{i} a_{j}, a_{i}=v, a_{i j}^{*}=v(v-1)$, получаем формулу

$$
\sigma_{N}^{2}\left(\alpha_{N}\right)=e^{-\alpha_{N} v}\left(1-\left(1+\alpha_{N} v\right) e^{-\alpha_{N} v}\right) .
$$

\section{3. Асимптотическая нормальность}

Для доказательства асимптотической нормальности $\mu_{0}(n, N)$ удобно воспользоваться теоремой 2 работы [5]. Эта теорема является переформулировкой теоремы 2 работы В.Г. Михайлова [8], которая, в свою очередь, обобщает теорему 2 работы С. Янсона [7]. 
Так же, как в работах [5], [6], полагаем

$$
\varphi=\varphi_{N}=\left[\frac{(1+\delta) \ln N}{\ln \ln N}\right], \quad 0<\delta<1 .
$$

Случайную величину $\mu_{0}(n, N)$ представим, используя $(13)$ и разбиение (19) множества $T(l)$, в виде

$$
\mu_{0}(n, N)=\mu_{1}(N)+\mu_{2}(N)+\mu_{3}(N)+\Delta_{\varphi},
$$

где

$$
\begin{aligned}
& \mu_{u}(N)=\sum_{l=0}^{\varphi-1} \sum_{t(l) \in T_{u}(l)} \xi_{l}\left(t_{1}, \ldots, t_{l}\right), \quad u=1,2,3 \\
& \left|\Delta_{\varphi}\right| \leqslant \Delta_{1}(\varphi)+\Delta_{2}(\varphi)+\Delta_{3}(\varphi) \\
& \Delta_{u}(l)=\sum_{t(l) \in T_{u}(l)}\left|\xi_{l}\left(t_{1}, \ldots, t_{l}\right)\right| .
\end{aligned}
$$

Так же, как в работе [6], проверяется, что в схеме серий $(2)-(3)$ при $n, N \rightarrow \infty$ случайная величина $\left(\mu_{0}(n, N)-\mu_{1}(N)\right) / \sqrt{\mathbf{D} \mu_{0}}$ стремится к 0 по верояности, если

$$
\sigma_{N}^{2}\left(\alpha_{N}\right) \geqslant \varepsilon>0
$$

Далее для величины $\mu_{1}(N)$ проверяются условия теоремы 2 работы [5] и устанавливается асимптотическая нормальность $\mu_{1}(N)$, а следовательно, и $\mu_{0}(n, N)$. При проверке условий теоремы 2 из [5] используется лемма 1. Рассуждения, проводимые по этой схеме, приводят к следующей теореме об асимптотической нормальности величины $\mu_{0}(n, N)$, определенной по последовательности (1).

Теорема 2. Если в схеме серий (2) - (3) выполнено условие (22), то при $n, N \rightarrow \infty$ распределение величинь

$$
\left(\mu_{0}(n, N)-\mathbf{E} \mu_{0}(n, N)\right) / \sqrt{\mathbf{D} \mu_{0}(n, N)}
$$

сходится к стандартному нормальному распределению.

Замечание. В случае, когда объем комплекта равен единице $(v=1)$, утверждение теоремы 2 превращается в теорему 3 работы [5].

Автор признателен рецензенту за полезные замечания. 


\section{Список литературы}

[1] Колчин В.Ф., Севастьянов Б.А., Чистяков В.П., Случайные размещения, Наука, Москва, 1976.

[2] Михайлов В.Г., "Сходимость к многомерному нормальному закону в равновероятной схеме размещения частиц комплектами", Матем. сб., 111(153):2 (1980), 163-186.

[3] Жуков Е.А., Чистяков В.П., "О схемах размещения комплектов, аппроксимируемых полиномиальной схемой", Матем. заметки, 25:3 (1979), 455-463.

[4] Тихомирова М.И., Чистяков В.П. Об асимптотике моментов числа непоявившихся sцепочек, Дискр. матем, 9:1 (1997), 12-29.

[5] Тихомирова М.И., Чистяков В.П., “Асимптотическая нормальность числа непоявившихся значений т-зависимых случайных величин", Математические вопросы криптографии, 2:1 (2011), 119-129.

[6] Тихомирова М.И., “Асимптотическая нормальность числа непоявившихся несплошных исходов независимых испытаний”, Дискр. матем., 21:2 (2009), 112-125.

[7] Janson S., "Normal convergence by higher semi-invariants with applications to sums of dependent random variables and random graphs", Ann. Probab., 16:1 (1988), 305-312.

[8] Михайлов В.Г., “Об одной теореме Янсона”, Теория вероятн. и примен., 36 (1991), 168-170.

[9] Сачков В.Н., "Случайные неравновероятные покрытия и функциональные уравнения", Труды по дискретной математике, 5 (2002), 205-218.

[10] Михайлов В.Г., “Оценка точности пуассоновской аппроксимации для числа пустых ячеек при размещении частиц комплектами", Труды МИАН, 282 (2013), 165-180.

[11] Ватутин В. А., Михайлов В. Г., "Предельные теоремы для числа пустых ячеек в равновероятной схеме размещения частиц комплектами", Теория вероятн. и примен., 28:4 (1982), 684-692. 